\title{
Cellular homeostasis, implantation window and unexplained infertility: role of phosphatase and tensin homolog deleted on chromosome 10
}

\author{
Annu Makker ${ }^{1 *}$, Madhu Mati Goel², Kumari Manu³, Renu Makker ${ }^{4}$
}

\begin{abstract}
${ }^{1}$ Department of Biochemistry, Prasad Institute of Medical Sciences, Lucknow, Uttar Pradesh, India ${ }^{2}$ Department of Pathology, King George's Medical University, Lucknow, Uttar Pradesh, India ${ }^{3}$ Department of Pathology, Prasad Institute of Medical Sciences, Lucknow, Uttar Pradesh, India ${ }^{4}$ Makker IVF Centre and Infertility Clinic, Lucknow, Uttar Pradesh, India
\end{abstract}

Received: 27 April 2019

Accepted: 05 June 2019

\section{*Correspondence:}

Dr. Annu Makker,

E-mail: annumakker@gmail.com

Copyright: (c) the author(s), publisher and licensee Medip Academy. This is an open-access article distributed under the terms of the Creative Commons Attribution Non-Commercial License, which permits unrestricted non-commercial use, distribution, and reproduction in any medium, provided the original work is properly cited.

\begin{abstract}
Background: Balance between endometrial cell proliferation and apoptosis is crucial for successful embryo implantation. PTEN (phosphatase and tensin homolog deleted on chromosome 10), a pro-apoptotic factor, is proposed to be one of the signaling proteins through which estrogen and progesterone act to affect cellular homeostasis. Although reports in literature have suggested role of PTEN in regulating endometrial cell proliferation and apoptosis during window of implantation, its involvement in women with unexplained infertility is not clear. In the present study, we examined expression, cellular distribution and activation status of PTEN, cell proliferation, and apoptosis in midsecretory endometrium from women with unexplained infertility as compared to fertile controls.

Methods: Endometrial biopsies from infertile $(n=11)$ and fertile women $(n=22)$ were used for immunohistochemical evaluation of PTEN, phospho-PTEN and Ki67. Terminal deoxynucleotidyl transferase-mediated deoxyuridine triphosphate nick end labeling assay was performed for detection of apoptotic cells.

Results: Biopsies from infertile women as compared to fertile controls demonstrated statistically significant: i) decrease in nuclear PTEN ( $\mathrm{P}<0.001)$, increase in nuclear phospho-PTEN $(\mathrm{P}<0.05)$, increase in nuclear and cytoplasmic phospho-PTEN/PTEN ratio ( $\mathrm{P}<0.001$ and $\mathrm{P}<0.05$ respectively) in endometrial stroma, ii) increase in cytoplasmic phospho-PTEN $(\mathrm{P}<0.001)$ and phospho-PTEN/PTEN ratio $(\mathrm{P}<0.05)$ in glandular epithelium $(\mathrm{GE})$, iii) increase in Ki67 labeling in GE $(\mathrm{P}<0.01)$ and stroma $(\mathrm{P}<0.05)$ and, iv) decrease in $(\mathrm{P}<0.001)$ apoptosis.

Conclusions: Altered PTEN expression and associated modulation in cellular homeostasis during the implantation window might contribute to mechanism underlying unexplained infertility.
\end{abstract}

Keywords: Apoptosis, Cell proliferation, Endometrium, Implantation window, PTEN, Unexplained infertility

\section{INTRODUCTION}

Infertility is an important condition in reproductive medicine, which, besides being a medical problem has considerable psychological morbidity and imposes substantial economic burden. ${ }^{1}$ Female infertility occurs in about $37 \%$ of all infertile couples. In $15-30 \%$ of infertile women, no apparent cause of infertility is found during infertility work-up and the type of infertility is categorized as unexplained infertility. ${ }^{2}$ Although, Assisted Reproductive Technology (ART) has given a new ray of hope for such couples, success rate is still not more than $30 \%$ in best of ART laboratories. ${ }^{3}$

Implantation failure is one of the key reasons underlying low success rate of ART. Successful implantation depends upon two critical factors, first appropriate embryo development to the blastocyst stage, and, second 
synchronous development of endometrium which is receptive to blastocyst implantation. The restricted time period during which the uterine environment is conducive to blastocyst acceptance and subsequent implantation is referred to as the "receptivity period" or the "window of implantation". Studies in humans with donor embryos have shown that endometrial receptivity is limited to day's 6-10 post ovulation (i.e. days 20-24 or midsecretory phase of a normal 28-30 days menstrual cycle). ${ }^{4}$ Endometrial development resulting in endometrial receptivity during the window of implantation requires collaboration of an extremely large number of different factors, including maternal steroid hormones, growth factors, and cytokines in a paracrine manner. Various signaling proteins viz. ERKs, PI3K/Akt/mTOR etc. have also been shown to be involved in the coordination and integration of key events during the implantation window. ${ }^{5}$

Reports in literature have shown that a balance between endometrial cell proliferation and apoptosis is crucial to maintain cellular homeostasis during implantation window. Normally, apoptosis of endometrial cells is significantly higher during the period of uterine receptivity, allowing successful invasion of blastocyst into the stroma and its subsequent implantation. ${ }^{6}$ Alterations in pro- and anti-apoptotic events in the secretory endometrium have been suggested to be involved in implantation disorders and could be cause of in vitro fertilization (IVF) failure in infertile women with endometriosis, tubal factor, or polycystic ovary syndrome (PCOS). ${ }^{7}$ Vatansever et al, reported reduced apoptosis and altered $\mathrm{Bcl}: \mathrm{Bax}$ ratio in endometrium of women with unexplained infertility as compared to fertile women. ${ }^{8}$

PTEN (phosphatase and tensin homolog deleted on chromosome 10), a pro-apoptotic factor and tumor suppressor gene has been suggested to be one of the signaling proteins through which estrogen and progesterone act to affect endometrial cell proliferation and apoptosis. ${ }^{9,10}$ PTEN negatively regulates the activity of the PI3K/Akt pathway by dephosphorylating PIP3 (phosphatidylinositol 3,4,5-triphosphate) to produce PIP2 (phosphatidylinositol 4,5-bisphosphate), thereby preventing phosphorylation (activation) of Akt. Activated Akt has been implicated in various cellular functions including growth, proliferation, survival, apoptosis and migration. ${ }^{11}$ Phosphorylation of PTEN at its C-terminal domain causes its inactivation and prevents degradation. ${ }^{12}$

Previous studies have reported novel roles for PTEN in mammalian uterus and its necessity for proper trophoblast invasion and decidual regression. ${ }^{13}$ Antsiferova et al, observed that synthesis of the proapoptotic factor, PTEN, was considerably increased in endometrium of women with tubal factor infertility. They associated the high level of PTEN mRNA expression with preparation of endometrium for embryo implantation and to success of IVF treatment in these women. Further, lower PTEN mRNA expression in the endometrium was associated with lower IVF success rate. $^{7}$

Although the role of PTEN in regulating cellular homeostasis during embryo implantation is apparent, its involvement in endometrium from women with unexplained infertility during the window of implantation is yet not clear. Hence, in the present study, we have examined and compared expression, cellular distribution, and activation status of PTEN in midsecretory endometrium from women with unexplained infertility with that from fertile women serving as study controls. We also evaluated alterations in cellular homeostasis by assessing proliferative activity through immunohistochemical localization of Ki67 antigen and apoptotic cell death by terminal deoxynucleotidyl transferase-mediated deoxyuridine triphosphate nick end labeling (TUNEL) assay.

\section{METHODS}

\section{Participants}

The study included fertile women volunteers $(n=11)$ undergoing tubal sterilization or reversal of tubal sterilization and women with unexplained infertility $(n=22)$. Study period was from November, 2017 to February, 2019. Human tissue was used for research only after informed consent and approval by Institutional Ethics Committee. The study controls were healthy, fertile women aged between 27 and 38 years (mean \pm standard error of the mean (SEM): $33.45 \pm 1.32$ years), with mean parity of 2.06 (range: 1-3) and no history of infertility or abortions. The mean age of infertile women was 31.04 \pm 0.81 years (range: 25-36) and the mean duration of infertility was $6.14 \pm 0.74$ years. Each infertile woman underwent complete infertility work-up at the Makker IVF Centre and Infertility clinic, Lucknow. Criteria for diagnosis of unexplained infertility included: i) infertility for at least 2 years; ii) normal history and physical examination; iii) adequate coital frequency; iv) three normal semen analyses; v) regular monthly menstrual cycles with biphasic BBT and luteal phase $\geq 12$ days; vi) adequate cervical mucus and a normal post coital test; vii) normal luteinizing hormone, follicle stimulating hormone, prolactin, testosterone, and progesterone $(\mathrm{P})$; viii) normal hysterosalpingography and laparoscopic findings. ${ }^{2}$

All volunteers had regular menstrual cycles every 28-32 days. All study participants underwent transvaginal ultrasonographic monitoring of ovulation commencing from days 8-10 of menstrual cycle using a 3.5 to $8.5 \mathrm{MHz}$ vaginal transducer (Shimadzu SBU 350, Japan). The day of ovulation was the day of maximum follicular enlargement and was followed by sudden disappearance or filling in of follicle showing clear demarcation of walls and intrafollicular echoes. The midsecretory phase ( +6 to +10 days post ovulation) was confirmed by histologic dating of the endometrium and by serum $\mathrm{P}$ levels. 
Menstrual cycles of all participants were ovulatory based on mid luteal serum $\mathrm{P}$ values of $>10 \mathrm{ng} / \mathrm{ml}$.

\section{Endometrial samples and histological dating}

Endometrial biopsies were obtained from the uterine fundus from all participants during the midluteal phase of the menstrual cycle using a standard endometrial suction curette (Gyne'tics, Belgium). Samples were fixed in 4\% buffered formaldehyde for 24 hours, embedded in paraffin and sectioned at $4 \mu \mathrm{m}$ and mounted on 3aminopropyl triethoxysilane (APTES)-coated glass slides and processed for histologic dating and immunohistochemical studies.

Histologic dating for each biopsy was performed by the same pathologist (K.M.) following the Noyes criteria (1950). ${ }^{14}$ Histologically proven midsecretory biopsies were included in the study.

\section{Immunohistochemistry}

For immunohistochemical localization of PTEN, p-PTEN and Ki67, APTES coated slides with sections were incubated at $60^{\circ} \mathrm{C}$ for 1 hour, deparaffinized and the rehydrated. For antigen retrieval, sections were placed in boiling Tris-EDTA buffer ( $\mathrm{pH}$ 9.0) for $15 \mathrm{~min}$ for antibodies against p-PTEN (ab131107; Abcam) and Ki67 (IS626; DAKO, Denmark); and in boiling EDTA buffer ( $\mathrm{pH}$ 8.0) for $15 \mathrm{~min}$ for antibody against PTEN (clone 6H2.1, \#04-035; Millipore, Darmstadt, Germany). Detection was performed using Novolink Min Polymer Detection System (Novacastra; Leica Biosystems, Newcastle Lt, United Kingdom).

The chromogen, 3, 3'-diaminobenzidine tetra hydrochloride was used for visualizing the immunoreaction. Sections were counterstained with hematoxylin, dehydrated with a series of graded ethanol washes, mounted with DPX (dibutyl phthalate xylene) mountant (Rankem, India), and cover slipped for bright field microscopy. Positive controls for each antibody were selected according to manufacturer's recommendations. Negative controls were sections processed without incubation with primary antibody.

\section{Semi quantitative scoring of immunohistochemical staining}

All samples were evaluated independently by 2 observers (AM and KM). In case of discrepancy, the sections were reassessed and a consensus was reached. The surface or luminal epithelium (SE), glandular epithelium (GE) and stroma were assessed for localization of stain (nuclear or cytoplasmic) and the intensity and proportion of cells stained were scored on a scale of 0 to 4 as described previously. ${ }^{15}$ The immunointensity and immunopositivity scores were multiplied giving results ranging from 0 to 16.
Ki67 labeling index was calculated as the percentage of cells stained with Ki67 antibody (by evaluating approximately 1000 nuclei from SE, GE and stromal cells).

\section{The TUNEL assay}

Detection of apoptotic cells by TUNEL assay and the criteria for evaluating apoptotic cells was as described previously. ${ }^{15}$ For quantification of apoptotic cells, TUNEL stained sections were evaluated independently by $\mathrm{AM}$ and $\mathrm{KM}$, and the apoptotic index (number of apoptotic cells per 1000 cells) was determined.

\section{Statistical analysis}

One way analysis of variance (unstacked) for comparison of means between 2 groups was used to analyze the immunohistochemistry and apoptosis data using the Minitab 13.20 statistical software (Minitab Inc. State College, Pennsylvania). $\mathrm{P}$ value of $<0.05$ was considered as statistically significant.

\section{RESULTS}

\section{PTEN and p-PTEN}

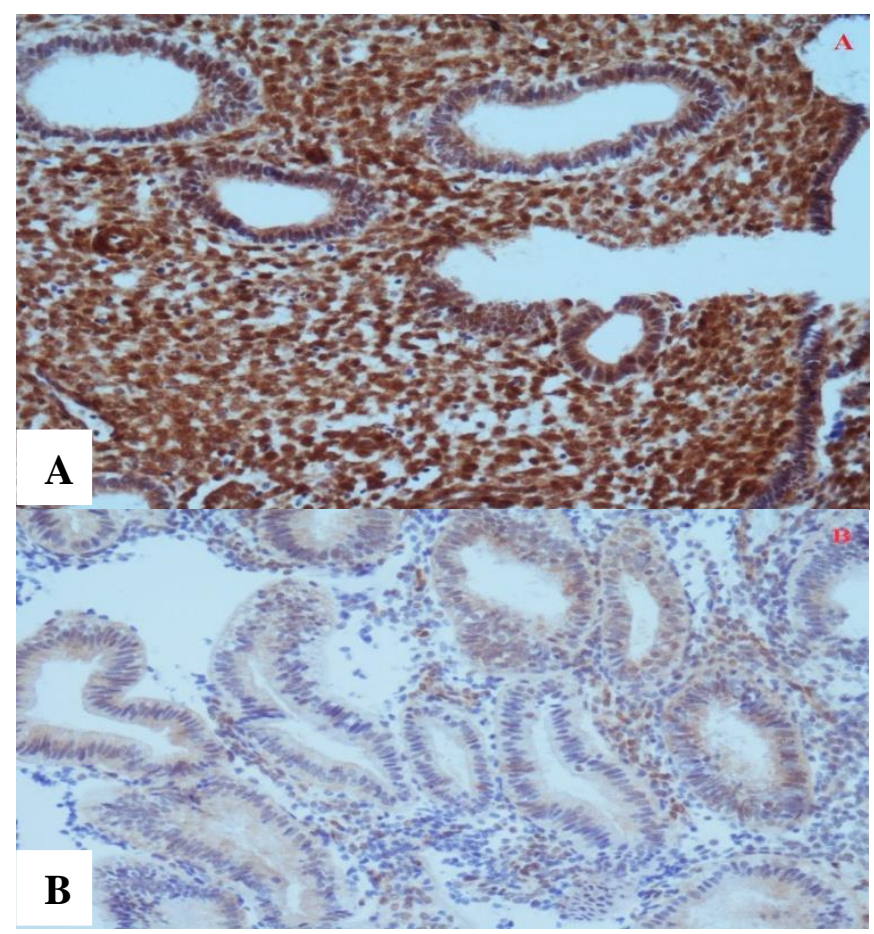

Figure 1: (A): Photomicrograph of histological section of midsecretory endometrium obtained from a fertile woman showing high cytoplasmic and nuclear immunohistochemical staining for PTEN. Original magnification $x$ 200. (B): Photomicrograph of histological section of midsecretory endometrium from a woman with unexplained infertility showing decrease in PTEN staining as compared to fertile control. Original magnification x 200. 
Table 1: Expression of PTEN, p-PTEN, Ki67 and apoptosis in midsecretory endometrium from women with unexplained infertility and fertile women.

\begin{tabular}{|c|c|c|c|c|}
\hline \multirow[b]{2}{*}{ Protein } & \multicolumn{2}{|c|}{ Fertile control } & \multicolumn{2}{|c|}{ Infertile women } \\
\hline & Cytoplasmic & Nuclear & Cytoplasmic & Nuclear \\
\hline \multicolumn{5}{|l|}{ PTEN } \\
\hline LE & $6.65 \pm 3.53$ & $6.05 \pm 3.93$ & $4.47 \pm 3.21$ & $3.82 \pm 4.60$ \\
\hline GE & $7.91 \pm 3.33$ & $7.41 \pm 3.81$ & $5.8 \pm 2.52$ & $5.16 \pm 3.48$ \\
\hline Stroma & $8.09 \pm 1.86$ & $10.27 \pm 2.64$ & $6.00 \pm 3.38$ & $5.93 \pm 3.10^{* * *}$ \\
\hline \multicolumn{5}{|l|}{ p-PTEN } \\
\hline LE & $5.60 \pm 2.07$ & $4.00 \pm 2.59$ & $7.05 \pm 3.60$ & $3.88 \pm 2.73$ \\
\hline GE & $5.64 \pm 2.57$ & $4.77 \pm 3.13$ & $10.11 \pm 3.00 * * *$ & $4.02 \pm 3.28$ \\
\hline Stroma & $6.41 \pm 2.47$ & $4.82 \pm 1.66$ & $7.71 \pm 2.99$ & $7.64 \pm 4.21 *$ \\
\hline \multicolumn{5}{|l|}{ p-PTEN/PTEN } \\
\hline LE & $1.04 \pm 0.62$ & $0.85 \pm 1.10$ & $2.76 \pm 2.90$ & $1.17 \pm 1.35$ \\
\hline GE & $0.81 \pm 0.49$ & $0.68 \pm 0.50$ & $1.99 \pm 0.98 * *$ & $0.90 \pm 0.81$ \\
\hline Stroma & $0.81 \pm 0.33$ & $0.49 \pm 0.17$ & $1.44 \pm 0.99 *$ & $1.23 \pm 0.47 * * *$ \\
\hline \multicolumn{5}{|l|}{ Ki67 } \\
\hline LE & & $5.15 \pm 6.61$ & & $6.45 \pm 5.93$ \\
\hline GE & & $3.15 \pm 2.15$ & & $16.48 \pm 14.42 * *$ \\
\hline Stroma & & $19.92 \pm 12.49$ & & $35.33 \pm 24.88^{*}$ \\
\hline \multicolumn{5}{|l|}{ Apoptotic index } \\
\hline LE+GE+Stroma & & $8.86 \pm 2.85$ & & $2.80 \pm 1.90 * * *$ \\
\hline
\end{tabular}

Immunohistochemical staining for PTEN was cytoplasmic and/or nuclear in the epithelial and stromal compartments of the midsecretory endometrial biopsies obtained from women in both groups (Figures 1A and 1B). Nuclear PTEN immunoscore in the stroma of infertile women $(5.93 \pm 3.10)$ was significantly lower $(\mathrm{P}<0.001)$ than that observed in the stroma of fertile controls (10.27 \pm 2.64$)$. No significant difference in immunostaining was observed in the SE and GE compartments of endometrium from both infertile and fertile women (Table 1).

Immunostaining for PTEN phosphorylated at the serine 380 and threonine 382/383residues was cytoplasmic and/or nuclear in the SE, GE and stroma (Figures $2 \mathrm{~A}$ and 2B). Biopsies from infertile women as compared to fertile controls showed significantly higher immunoscore for cytoplasmic p-PTEN in the GE $(10.11 \pm 3.00$ vs. $5.64 \pm 2.57 ; \mathrm{P}<0.001)$ and nuclear $\mathrm{p}-\mathrm{PTEN}$ in the stroma (7.64 \pm 4.21 vs. $4.82 \pm 1.66 ; \mathrm{P}<0.05)$. No significant difference was observed in the surface epithelium (Table $1)$.

The cytoplasmic and nuclear p-PTEN/PTEN ratio was evaluated for both epithelial and stromal compartments. Biopsies from infertile women as compared to fertile controls showed significantly higher cytoplasmic pPTEN/PTEN ratio in the GE $(1.99 \pm 0.98$ vs. $0.81 \pm 0.49 ; \mathrm{P}$ $<0.05)$ cytoplasmic $(1.44 \pm 0.99$ vs. $0.81 \pm 0.33$; $\mathrm{P}<0.05)$ and nuclear $(1.23 \pm 0.47$ vs. $0.49 \pm 0.17$; $\mathrm{P}<0.001) \mathrm{p}$ PTEN/PTEN ratio in the stroma. No significant difference was observed in the surface epithelium (Table 1).

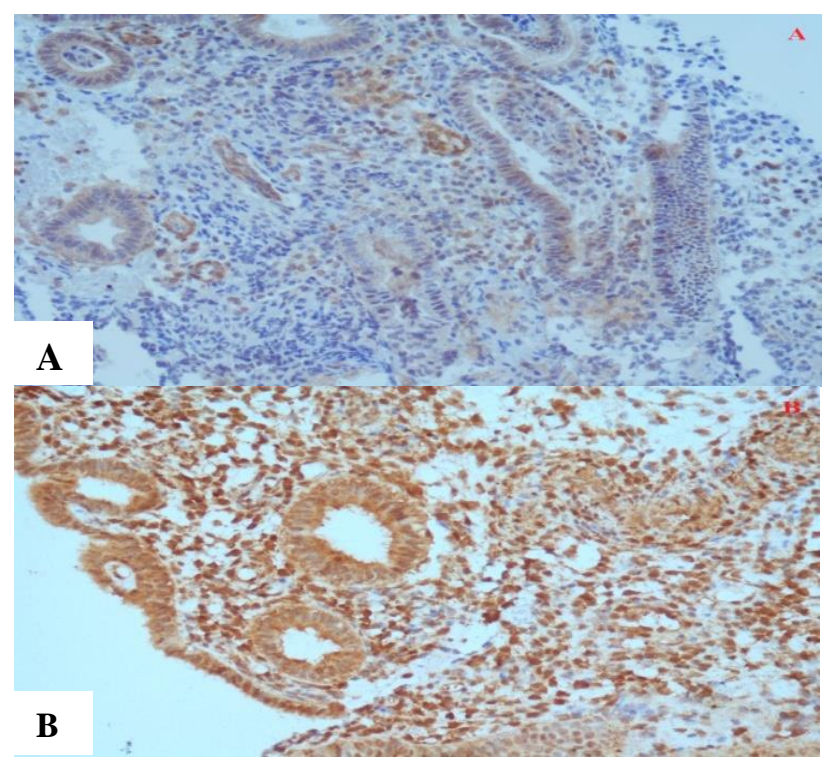

Figure 2: (A): Photomicrograph of histological section of midsecretory endometrium from a fertile woman showing weak cytoplasmic and nuclear immunohistochemical staining for phospho-PTEN. Original magnification x 200, (B): Photomicrograph of histological section of midsecretory endometrium from a woman with unexplained infertility showing higher phospho-PTEN staining as compared to fertile control. Original magnification x 200 . 


\section{Ki67}

An increase in Ki67 immunopositivity was observed in the midsecretory endometrium from infertile women as compared to fertile controls (Figures 3A and 3B). The GE $(16.48 \pm 14.42$ vs. $3.15 \pm 2.15 ; \mathrm{P}<0.01)$ and Stroma $(35.33 \pm 24.88$ vs. $19.92 \pm 12.49 ; \mathrm{P}<0.05)$ of infertile group showed significantly higher Ki67 labeling index as compared to controls. However, no significant difference in number of Ki67 positive nuclei was observed in the surface epithelium (Table 1).

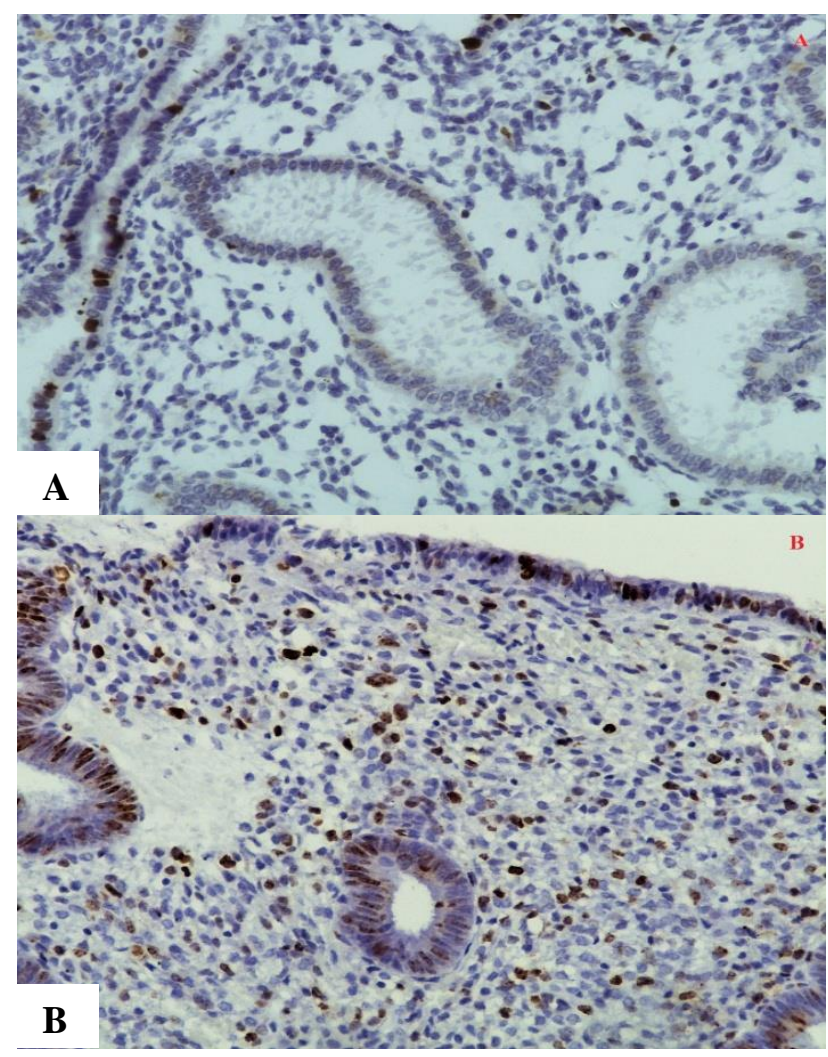

Figure 3: (A): Photomicrograph of histological section of midsecretory endometrium from a fertile woman showing immunohistochemical staining for Ki67. Original magnification x 200. (B): Photomicrograph of histological section of midsecretory endometrium from a woman with unexplained infertility showing significantly higher number of Ki67 positive nuclei as compared to fertile control. Original magnification x 200 .

\section{Apoptosis}

The apoptotic index in the midsecretory endometrium of infertile women (Figure 4B) was significantly lower $(2.80 \pm 1.90$ vs. $8.86 \pm 2.85$; $\mathrm{P}<0.001$; Table 1$)$ as compared to the fertile controls (Figure 4A). Maximum apoptotic cells were observed in the glandular epithelium, much less in the stroma and were nearly absent in the surface epithelium.

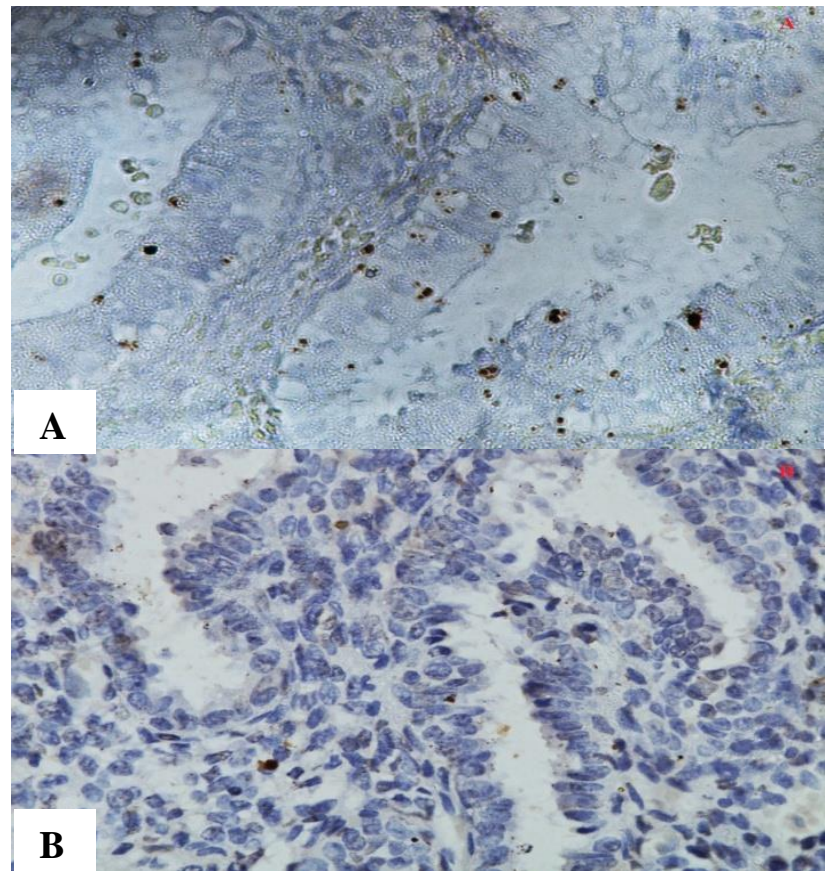

Figure 4: (A): Photomicrograph of histological section of midsecretory endometrium from a fertile woman showing large number of apoptotic cells. Original magnification x 400. (B): Photomicrograph of

histological section of midsecretory endometrium

from a woman with unexplained infertility showing very few apoptotic cells. Original magnification x 400 .

\section{DISCUSSION}

PTEN, one of the most studied tumor suppressor genes, is known to play significant role in controlling various cellular processes including cell proliferation and growth, inducing cell cycle arrest, programming apoptosis, regulation of cell adhesion, migration and differentiation. ${ }^{11}$ It has been reported that endometrial PTEN expression changes throughout the menstrual cycle, and that regulation of PTEN expression by ovarian steroids helps to protect balance between endometrial proliferative and apoptotic activities, events crucial for endometrial receptivity for embryo implantation. ${ }^{10}$ Although the role of PTEN in regulating cellular homeostasis during embryo implantation is explicable from available studies in infertile women with tubal factor and endometriosis, its involvement in endometrium from women with unexplained infertility during the window of implantation is yet not clear. ${ }^{7}$

In the present study, we evaluated immunohistochemical expression, cellular distribution and activation status of PTEN in midsecretory endometrium from women with unexplained infertility as compared to fertile controls. Ki67 labeling index (a marker of cell proliferation) and apoptosis were examined to have insight into cellular homeostasis during implantation window in these women. We observed significantly lower nuclear PTEN expression in endometrial stroma of infertile women as 
compared to fertile controls. Immunostaining for PTEN phosphorylated at the serine 380 and threonine 382/383 residues revealed activation status of PTEN. Increased nuclear p-PTEN expression as well as p-PTEN/PTEN ratio was seen in endometrial stroma of biopsies from infertile women as compared to fertile controls, suggesting loss of biological activity of PTEN.

Although no information, to the best of our knowledge, is available on p-PTEN/PTEN ratio in women with unexplained infertility, study in women with tubal factor infertility demonstrated that pregnancy achievement was associated with maximal level of the PTEN mRNA expression in the endometrium and suggested that PTEN is essential for endometrial receptivity and successful implantation. $^{7}$ Laguë et al, studied implantation in transgenic mice lacking PTEN gene in myometrial and stromal/decidual cells. Loss of PTEN was suggested to result in altered implantation and/or uterine glandular function and henceforth contribute to infertility/fetal loss in these mice. ${ }^{13}$

In a recent study, low level laser therapy (LLLT) was shown to significantly enhance endometrial receptivity by promoting healthy cell growth and proliferation. Significantly greater PTEN expression was observed in groups that were lasered in comparison to the ones that received no laser. The results suggested that LLLT may serve as a new complementary therapy in improving endometrial receptivity and regeneration by inducing up regulation of PTEN gene expression. ${ }^{16}$

In the present study, we found significantly higher number of Ki67 immunopositive nuclei in GE and stroma of midsecretory endometrial biopsies from infertile women as compared to fertile controls, suggesting increased proliferative activity in women with unexplained infertility during implantation window.

Further, we observed significant reduction in total apoptotic index in endometrium of infertile women as compared to fertile controls. Pertinently, a previous study has reported reduced amount of apoptotic cells along with changed $\mathrm{Bcl}$ : Bax ratio in endometrium of women with unexplained infertility as compared to fertile women. An association of high endometrial apoptosis during the period of receptivity and IVF success has been reported in women with endometriosis. ${ }^{7}$ Midsecretory phase endometrium from infertile women with PCOS has been reported to exhibit scarce or absent apoptosis along with increased proliferative activity. The altered apoptotic activity and cell proliferation was suggested to be regulated by the PI3K/Akt signaling pathway and may be accountable for implantation failures in this patients. ${ }^{17}$

Recently, microRNAs have been reported to play a role in endometrial receptivity and decidualization. ${ }^{18}$ Shen et al, proposed PTEN to be target gene of mmu-microRNA200a. Normally the expression of microRNA-200a in mouse endometrial stromal cells is downregulated during the period of receptivity. Injection of the uterine horn with mmu-miR-200a lentivirus resulted in decreased implantation rate which was attributed partly to cell proliferation and apoptosis regulation via PTEN, supporting thereby role of PTEN in endometrial receptivity. ${ }^{19}$

\section{CONCLUSION}

To conclude, our results demonstrate that altered expression of PTEN and the associated modulation in cellular homeostasis during the window of implantation might contribute to mechanism underlying unexplained infertility. Further studies in a larger population are required to ascertain the prospect of selective targeting of PTEN as an alternative treatment for unexplained infertility and also for improving results of ART in this category of infertile women.

\section{ACKNOWLEDGMENTS}

Authors would like to thank Dr. Geetanjali Mishra and Dr. Abnish Gautam for their invaluable support during the preparation of this manuscript. Also acknowledge the inputs by Mr. Rehan Ali.

\section{Funding: No funding sources}

Conflict of interest: None declared

Ethical approval: The study was approved by the Institutional Ethics Committee

\section{REFERENCES}

1. Edelmann RJ, Connolly KJ. Psychological aspects of infertility. Br J Med Psychol. 1986;59:209-19.

2. Kamath MS, Deepti MK. Unexplained infertility: An approach to diagnosis and management. Curr Med Issues. 2016;14:94-100.

3. Pandian Z, Bhattacharya S, Vale L, Templeton A. In vitro fertilization for unexplained subfertility. Cochrane Database Syst Rev. 2012;4:1-42.

4. Makker A, Singh MM. Endometrial receptivity in relation to fertility, infertility and antifertility: Clincal assessment using structural, biochemical and molecular markers. Med Res Rev. 2006;26:699-746.

5. Makker A, Goel MM, Das V, Agarwal A. PI3K-AktmTOR and MAPK signaling pathways in polycystic ovarian syndrome, uterine leiomyomas and endometriosis: an update. Gynecol Endocrinol. 2012;28:175-81.

6. Antsiferova YS, Sotnikova NY. Apoptosis and endometrial receptivity: Relationship with in vitro fertilization treatment outcome. World $\mathrm{J}$ Obstet Gynecol. 2016;5:87-96.

7. Antsiferova YS, Sotnikova NY, Bogatova IK, Boitsova AV. Changes of apoptosis regulation in the endometrium of infertile women with tubal factor and endometriosis undergoing in vitro fertilization treatment. JBRA Assisted Reproduction. 2014;18:26. 
8. Vatansever HS, Lacin S, Ozbilgin MK. Changed $\mathrm{Bcl}:$ Bax ratio in endometrium of patients with unexplained infertility. Acta Histochem. 2005;107:345-55.

9. Mutter GL, Lin MC. Fitzgerald JT, kum JB, Eng C. Changes in endometrial PTEN expression throughout the human menstrual cycle. J Clin Endocrinol Metab. 2000;85:2334-8.

10. Guzeloglu-Kayisli O, Kayisli UA, AI-Rejjal R, Zheng W, Luleci G, Arici A. Regulation of PTEN (phosphatase and tensin homolog deleted on chromosome 10) expression by estradiol and progesterone in human endometrium. J Clin Endocrinol Metab. 2003;88:5017-26.

11. Song MS, Samena L, Pandolfi PP. The functions and regulation of the PTEN tumour suppressor. Nature Review Mol Cell Biol 2012;13:283-296.

12. Yang Z, Yuan XG, Chen J, Luo SW, Luo ZJ, Lu NH. Reduced expression of PTEN and increased PTEN phosphorylation at residue Ser380 in gastric cancer tissues: a novel mechanism of PTEN inactivation. Clin Res Hepatol Gastroenterol. 2013;37:72-9.

13. Laguë MN, Detmar J, Paquet M, Boyer A, Richards JS, Adamson SL, Boerboom D. Decidual PTEN expression is required for trophoblast invasion in the mouse. Am J Physiol Endocrinol. 2010;299:E936E945.

14. Noyes RW, Hertig AT, Rock J. Dating the endometrial biopsy. Fertil Steril. 1950;1:3-25.

15. Makker A, Goel MM, Nigam D, Mahdi AA, Das V, Agarwal A, et al. Aberrant Akt activation during implantation window in infertile women with intramural uterine fibroids. Reproductive Sci. 2018;25:1243-53.

16. El Faham DA, Elnoury MAH, Morsy MI. Has the time come to include low-level laser photobiomodulation as an adjuvant therapy in the treatment of impaired endometrial receptivity? Lasers Med Sci. 2018;33:55-62.

17. Avellaira C, Villavicencio A, Bacallao K. Expression of molecules associated with tissue homeostasis in secretory endometria from untreated women with polycystic ovary syndrome. Hum Reprod. 2006;21:3116-21.

18. Jimenez PT, Mendelson CR. The miR-200 family and its targets in implantation and decidualization. Reprod Sci. 2014;21:102A.

19. Shen LJ, He JL, Yang DH, Ding YB, Chen XM, Geng YQ, et al. Mmu-microRNA-200a overexpression leads to implantation defect by targeting phosphatase and tensin homolog in mouse uterus. Reprod Sci. 2013;20:1518-28.

Cite this article as: Makker A, Goel MM, Manu K, Makker R. Cellular homeostasis, implantation window and unexplained infertility: Role of phosphatase and tensin homolog deleted on chromosome 10. Int J Reprod Contracept Obstet Gynecol 2019;8:2754-60. 